\title{
Effect of Dialkyl Ammonium Cationic Surfactants on the Microfluidity of Membranes Containing Raft Domains
}

\author{
Makoto Uyama $^{1 *}$, Kaori Inoue ${ }^{1}$, Koichi Kinoshita ${ }^{1}$, Reiji Miyahara ${ }^{1}$, Hirokazu Yokoyama ${ }^{2}$ \\ and Minoru Nakano ${ }^{2}$ \\ ${ }^{1}$ Shiseido Global Innovation Center, 2-2-1 Hayabuchi, Tsuzuki-ku, Kanagawa 224-8558, JAPAN \\ ${ }^{2}$ Graduate School of Medicine and Pharmaceutical Sciences, University of Toyama, 2630 Sugitani, Toyama 930-0194, JAPAN
}

\begin{abstract}
It has been reported that a lot of receptors localize in lipid raft domains and that the microfluidity of these domains regulates the activation of these receptors. In this study, we focused on the lipid raft and in order to evaluate the physicochemical effects of surfactants on microfluidity of lipid membranes, we used liposomes comprising of egg-yolk L- $\alpha$-phosphatidylcholine, egg-yolk sphingomyelin, and cholesterol as a model of cell membranes containing raft domains. The microfluidity of the domains was characterized by fluorescence spectrometry using 1,6-diphenyl-1,3,5-hexatriene and 2-dimethylamino-6-lauroylnaphthalene. Among several surfactants, dialkylammonium-type cationic surfactants most efficiently increased the microfluidity. It is therefore concluded that (1) the electrostatic interaction between the cationic surfactant and eggPC/eggSM/cholesterol liposome could be important, (2) surfactants with alkyl chains more effectively inserted into membranes than those with acyl chains, and (3) cationic surfactants with lower $T_{\mathrm{m}}$ values have a greater ability to increase the fluidity.
\end{abstract}

Key words: di-alkyl cationic surfactant, liposome, microfluidity, fluorescence spectrometry, raft domain

\section{INTRODUCTION}

Receptor activity is a crucial step for signaling regulation. It has been reported that a lot of receptors localize in lipid raft domains and that the microfluidity of these domains regulates the activation of these receptors ${ }^{1-8}$. Among of them, for example, TRPM8 (transient receptor potential melastatin 8 ) is a thermo-sensitive cation channel, that is highly expressed in cold sensory neurons and is activated by cold stimuli (threshold: $\sim 25^{\circ} \mathrm{C}$ ) and cooling agents such as menthol(2-isopropyl-5-methylcyclohexanol) and icilin (1-(2-hydroxyphenyl)-4- (3-nitrophenyl) -3,6-dihydropyrimidin-2-one $)^{1-5)}$. Palao has reported that TRPM8 localizes in lipid raft microdomains ${ }^{1}$. The mentholand cold-mediated responses of TRPM8 are potentiated when association of the channel to the microdomains is prevented by methyl- $\beta$-cyclodextrin, which is a compound that disrupts lipid rafts by removing cholesterol from membranes. And Janes has concluded that lipid rafts are important to control protein interactions in resting and activated $\mathrm{T}$ cells, and that aggregation of rafts following receptor ligation is a general mechanism for promoting immune cell signaling $^{7)}$. Hence, in this study, we focused on the lipid raft and evaluated the physicochemical effects of surfactants on microfluidity of lipid membranes

We used liposomes consisting of egg-yolk L- $\alpha$ phosphatidylcholine (eggPC), egg-yolk sphingomyelin (eggSM), and cholesterol as models of cell membranes containing raft domains ${ }^{9,10)}$. The microfluidity of the membranes was characterized via the fluorescence anisotropy of 1,6-diphenyl-1,3,5-hexatriene (DPH $)^{11-20)}$ and fluorescence-generalized polarization of 2-dimethylamino-6-lauroylnaphtalene (Laurdan ${ }^{20-24)}$. Laurdan was also used for two-photon microscopy experiments on phospholipid multi-lamellar membranes.

\section{EXPERIMENTAL}

\subsection{Materials}

EggPC and 1,2-palmitoyl-sn-glycero-3-phosphocholine (DPPC) were purchased from NOF Corp. (Tokyo, Japan). EggSM, cholesterol, and dihexadecyl phosphate(DHP, Fig.

\footnotetext{
*Correspondence to: Makoto Uyama, Shiseido Global Innovation Center, 2-2-1 Hayabuchi, Tsuzuki-ku, Kanagawa 224-8558, JAPAN E-mail: makoto.uyama@to.shiseido.co.jp Accepted September 4, 2017 (received for review May 29, 2017) Journal of Oleo Science ISSN 1345-8957 print / ISSN 1347-3352 online http://www.jstage.jst.go.jp/browse/jos/ http://mc.manusriptcentral.com/jjocs
} 
(a)

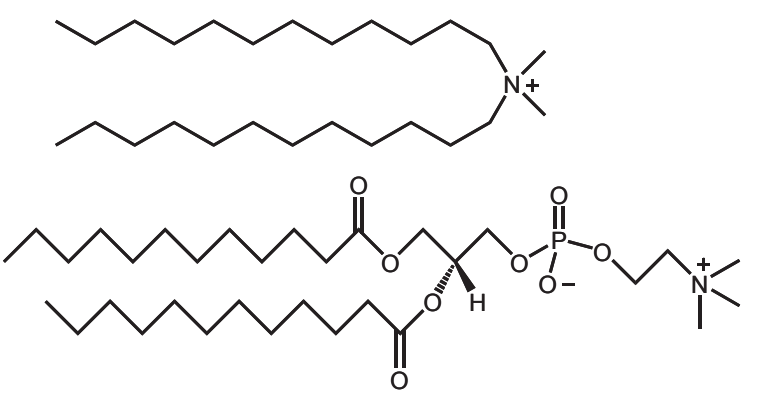

(c)

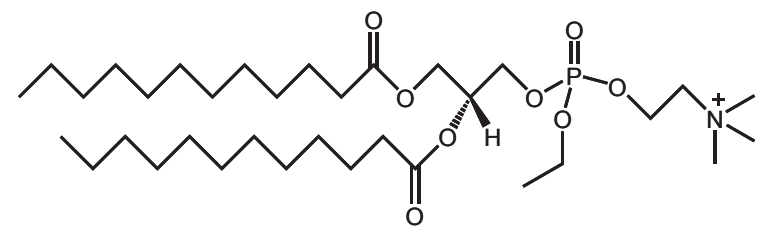

(d)

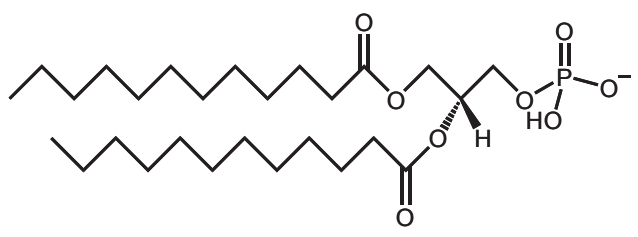

(e)

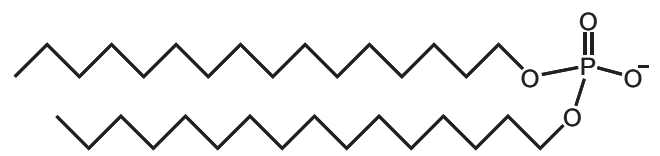

Fig. 1 Chemical structures of (a) DLAC, (b) DLPC, (c) ethyl-DLPC, (d)DLPA, and (e)DHP. Counter-ions are omitted.

1 (e)) were purchased from Sigma-Aldrich (St. Louis, MO, USA) . Dilauryl dimethyl ammonium chloride(DLAC, Fig. 1 (a) ) was purchased from Wako Pure Chemical Industries Ltd. (Osaka, Japan). Dilauryl dimethyl ammonium bromide (DLAB), dimyristyl dimethyl ammonium bromide (DMAB), dipalmityl dimethyl ammonium bromide (DPAB), and distearyl dimethyl ammonium chloride (DSAC) were purchased from Tokyo Chemical Industry Co., Ltd. (Tokyo, Japan) . 1,2-Dilauroyl-sn-glycero-3-phosphocholine (DLPC, Fig. 1 (b) ), 1,2-dilauroyl-sn-glycero-3-ethylphosphocholine (ethyl-DLPC, Fig. 1(c)), and 1,2-dilauroyl-sn-glycero3-phosphate (DLPA, Fig. 1(d)) were purchased from Avanti Polar Lipids (Alabaster, AL, USA). DPH and Laurdan were purchased from Invitrogen (Carlsbad, CA, USA).

\subsection{Liposome Preparation}

The eggPC/eggSM/cholesterol molar ratio of the liposomes was 7:6:6. Under this condition, liquid-ordered $\left(\mathrm{L}_{0}\right)$ and liquid-disordered $\left(\mathrm{L}_{\mathrm{d}}\right)$ phases are thought to coexist ${ }^{9,10}$. To prepare liposomes, the required amounts of chloroformmethanol solutions of eggPC, eggSM, cholesterol, and surfactants (if necessary) were mixed in a round-bottom glass flask. For the Laurdan-containing liposomes, a methanol solution of Laurdan was added at a Laurdan/lipid molar ratio of 1:500. The organic solvent was removed by evaporation, and the residue was dried overnight in vacuo. The dried lipids were dispersed in Tris buffer(i.e., $10 \mathrm{mM}$ Tris$\mathrm{HCl}$ and $300 \mathrm{mM}$ glucose, $\mathrm{pH}$ 7.4) by vortexing the mixture. After several freeze-thaw cycles, the suspension was extruded through a $100 \mathrm{~nm}$ pore-size polycarbonate filter. The mean radius of the liposome, as determined from dynamic light scattering (Photal FRAR-1000, Otsuka Electronic, Osaka, Japan), was ca. $65 \mathrm{~nm}$. Liposomes containing DPH were prepared by adding a DMSO solution of DPH to the liposomes at a DPH/lipid molar ratio of 1:100 and incubating them at $50^{\circ} \mathrm{C}$ for $1 \mathrm{~h}$.

\subsection{Fluorescence Measurements}

Fluorescence anisotropy experiments with DPH were performed on an F-4500 spectrofluorometer (Hitachi HighTechnology Corporation, Tokyo, Japan) at $32^{\circ} \mathrm{C}$. DPH, which is known to partition equally into $\mathrm{L}_{\mathrm{o}}(\mathrm{raft})$ and $\mathrm{L}_{\mathrm{d}}$ (non-raft) regions ${ }^{11,12)}$, was used to evaluate the fluidity of the lipid bilayers in the liposomes. Methanol solutions of each surfactant $(10 \mathrm{mg} / \mathrm{mL})$ were added to the liposome $(100 \mu \mathrm{M}$ of total lipids, $2 \mathrm{~mL})$. The excitation and emission wavelengths were 360 and $428 \mathrm{~nm}$, respectively. The steady-state anisotropy $(r)$ was calculated using the following the equation ${ }^{11-20)}$ :

$$
r=\frac{I_{\mathrm{VV}}-G I_{\mathrm{VH}}}{I_{\mathrm{VV}}+2 G I_{\mathrm{VH}}}
$$

where $I_{\mathrm{VV}}$ and $I_{\mathrm{VH}}$ are the measured fluorescence intensities with the excitation polarizer oriented vertically and the emission polarizer oriented vertically and horizontally, respectively. $G$ is the instrumental correction factor and equals to $I_{\mathrm{HV}} / I_{\mathrm{HH}}$, where $I_{\mathrm{HV}}$ and $I_{\mathrm{HH}}$ represent the intensities with the excitation polarizer oriented horizontally and the emission polarizer oriented vertically and horizontally, respectively.

The emission spectra of Laurdan were recorded from 400 to $550 \mathrm{~nm}$ with excitation at $350 \mathrm{~nm}$ on an RF-5300PC spectrofluorophotometer (Shimadzu Corporation, Kyoto, Japan) at $32^{\circ} \mathrm{C}$. Methanol solutions of each surfactant (10 $\mathrm{mg} / \mathrm{mL})$ were added to the liposome $(100 \mu \mathrm{M}$ of total lipids, $2 \mathrm{~mL}$ ). Laurdan displays emission maxima at 440 and 490 $\mathrm{nm}$ when the lipid bilayers form $\mathrm{L}_{\mathrm{o}}$ and $\mathrm{L}_{\mathrm{d}}$ phases, respectively ${ }^{20-24)}$. The shift of the emission maximum of Laurdan can be quantified via the generalized polarization (GP) value, which is calculated from the emission spectra as follows:

$$
\mathrm{GP}=\left(I_{440}-I_{490}\right) /\left(I_{440}+I_{490}\right),
$$

where $I_{440}$ and $I_{490}$ are the intensities of the emissions at 440 and $490 \mathrm{~nm}$, respectively.

\subsection{Two-photon Microscopy}

DPPC (500 nmol) and Laurdan $(1 \mathrm{nmol})$ dissolved in methanol were dropped onto slides and dried overnight in vacuo. Tris buffer $(200 \mu \mathrm{L})$ was added to the dried lipid films followed by $10 \mu \mathrm{L}$ of methanol surfactant solution(200 
$\mathrm{mg} / \mathrm{mL}$ ). Confocal microscopic images were recorded using a Radiance 2000 imaging system (BioRad, Jena, Germany) ${ }^{21,22,25)}$ at about $30^{\circ} \mathrm{C}$ with a thermo plate (MATS-55SFF2, Tokai Hit Co., Ltd, Shizuoka, Japan). The two-photon source is a tunable Ti-Sapphire laser (Mai Tai laser, Spectra Physics, Tokyo, Japan) with an excitation wavelength of $770 \mathrm{~nm}$. Interference filters were placed in the appropriate emission paths to isolate the 440 and $490 \mathrm{~nm}$ regions of the emission spectrum. Two simultaneous images $(256 \times 256$ pixels $)$ were obtained from the sample (image channel-1 and -2) and processed by applying the GP formula (Eq. (2)) to each pixel using Carl Zeiss ZEN software (Jena, Germany). A water-immersion $\times 60$ objective was used.

\section{RESULTS and DISCUSSION}

\subsection{Fluorescence anisotropy of DPH in eggPC/eggSM/ cholesterol liposome}

Figure 2 shows the variation of the steady-state fluorescence anisotropy $(r)$ of DPH in eggPC/eggSM/cholesterol liposome as a function of the concentration of surfactants added to the liposome. First, surfactants with two $\mathrm{C}_{12}$ chains and different head-groups (i.e., cationic, anionic, or twitterionic) were applied. It was verified that the fluorescence anisotropy was not affected by the addition of methanol without a surfactant (data not shown). As shown in Fig. 2, the anisotropy value decreased upon the addition of surfactants, which suggests an increase in the membrane fluidity. The order of the reduction of the anisotropy was DLAC $=$ DLAB $>$ DLPC $>$ DLPA $>$ ethyl-DLPC. Despite having different counter anions, there was no difference in the effects of DLAC and DLAB (data not shown). They both significantly increased the membrane fluidity. In contrast, ethyl-DLPC, which is another cationic surfactant, did not. The mean radius of the liposome increased from ca. 65 $\mathrm{nm}$ to $116 \mathrm{~nm}$ and $82 \mathrm{~nm}$ after the addition of $2.0 \times 10^{-2}$ wt\% DLAC and ethyl-DLPC, respectively; this implies that

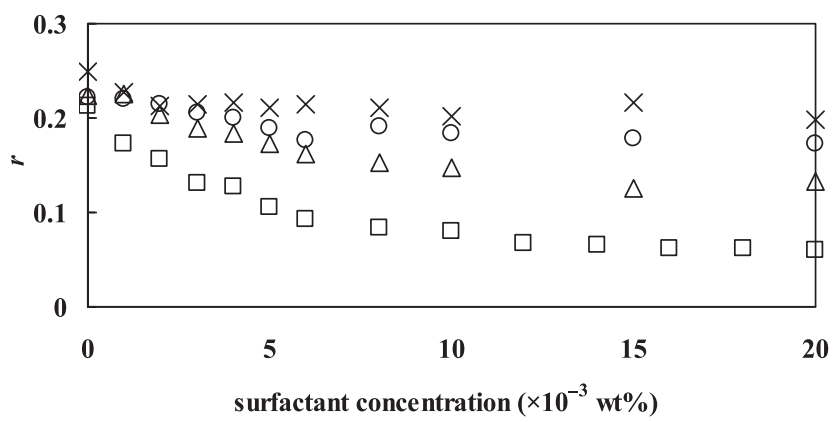

Fig. 2 Steady-state fluorescence anisotropy of DPH in eggPC/eggSM/cholesterol liposome at $32^{\circ} \mathrm{C}$ as a function of the wt \% concentration of ethyl-DLPC (crosses), DLPA (circles), DLPC (triangles), and DLAC (squares).

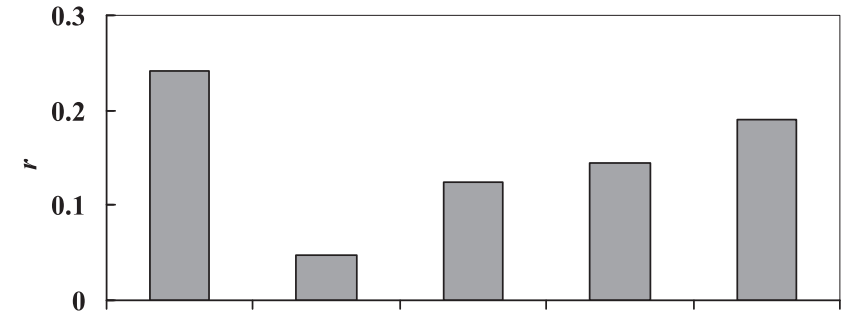

(a)

(b)

(c)

(d)

(e)

Fig. 3 Steady-state fluorescence anisotropy of DPH in (a) eggPC/eggSM/cholesterol (7:6:6) liposome and eggPC/eggSM/cholesterol (7:6:6)/surfactant (4:6) liposome at $32^{\circ} \mathrm{C}$. The surfactants are (b) DLAC, (c) ethyl-DLPC, (d)DLPC, and (e)DLPA.

ethyl-DLPC is integrated into the lipid membrane to a lesser extent than DLAC.

DPH anisotropy experiments were conducted on liposomes that had been mixed with surfactants before liposome preparation. The results are shown in Fig. 3. The order of the reduction of the anisotropy was DLAC $>$ ethylDLPC $>$ DLPC $>$ DLPA. The data again shows the high potential of DLAC to increase the membrane fluidity. The total lipids (eggPC/eggSM/cholesterol = 7:6:6) /surfactant molar ratio was $4: 6$, which corresponds to ca. $5.0 \times 10^{-3}$ wt \% surfactant for the experiments shown in Fig. 2. As the $r$ values for DLAC were comparable under both conditions, DLAC molecules are considered to be efficiently integrated when added to the liposome. The prominent difference between Figs. 2 and 3 is that the membrane containing 60 mol\% ethyl-DLPC had a significantly lower $r$ value than that containing DLPC or DLPA. Although ethyl-DLPC is capable of increasing the membrane fluidity, these results suggest that it is not readily incorporated into the membranes when added to liposomes.

Fluorescence anisotropy experiments were also conducted using surfactants with longer $\left(\mathrm{C}_{16}\right)$ hydrocarbon chains, i.e., DPAB (cationic), DHP(anionic), and DPPC (zwitterionic). As shown in Fig. 4, the $r$ value decreased

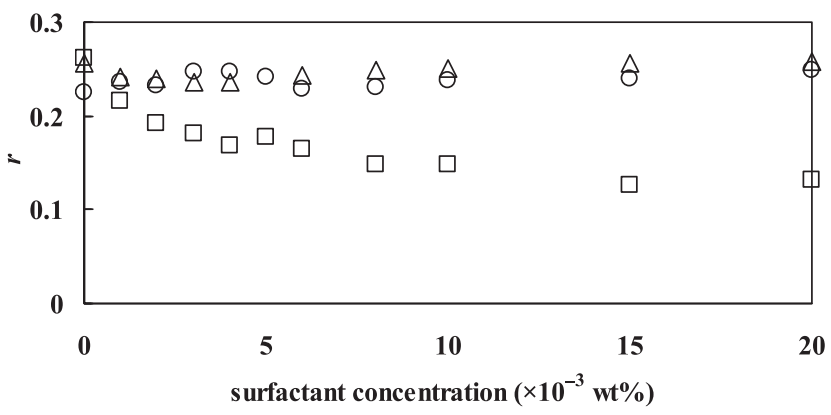

Fig. 4 Steady-state fluorescence anisotropy of DPH in eggPC/eggSM/cholesterol liposome at $32^{\circ} \mathrm{C}$ as a function of the wt \% concentration of DHP (triangles), DPPC (circles), and DPAB (squares). 


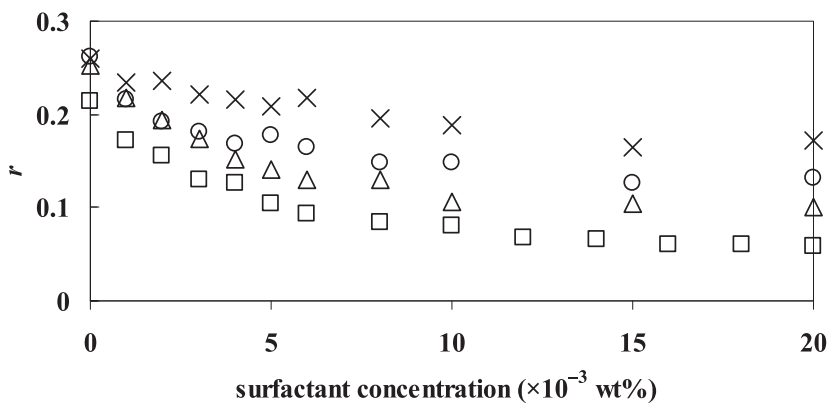

Fig. 5 Steady-state fluorescence anisotropy of DPH in eggPC/eggSM/cholesterol liposome at $32^{\circ} \mathrm{C}$ as a function of the wt $\%$ concentration of DSAC (crosses), DPAB (circles), DMAB (triangles), and DLAC (squares).

with the addition of DPAB to the liposomes, and increased with the addition of DHP and DPPC. The structure of DHP is similar to that of DPAB in that they have alkyl (not acyl) chains, however, they differ in electric charge. DHP has been reported to form bilayers independently and in mixtures with $\mathrm{PC} /$ cholesterol $^{26-31}$. The mean radius of the liposome after the addition of $2.0 \times 10^{-2}$ wt $\%$ DHP was 141 $\mathrm{nm}$, which is larger than that after the addition of $2.0 \times 10^{-2}$
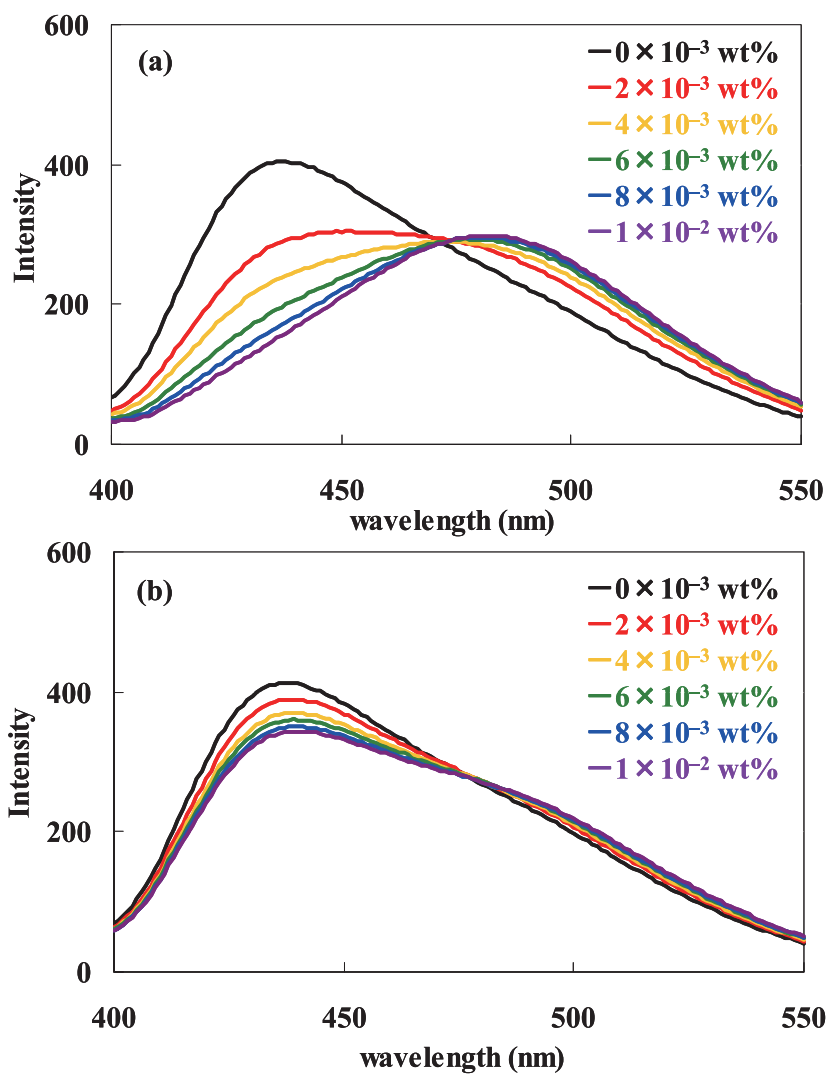

Fig. 6 Changes in the emission spectra of Laurdan in eggPC/eggSM/cholesterol liposomes at $32^{\circ} \mathrm{C}$ upon the addition of (a) DLAC and (b) ethyl-DLPC. wt\% DPAB (84 nm), suggesting that the DHP molecules are more integrated into the liposome. These results suggest that DHP does not increase the membrane fluidity. The gel-liquid crystalline phase transition temperature $\left(T_{\mathrm{m}}\right)$ of $\mathrm{DHP}\left(65^{\circ} \mathrm{C}\right)^{27-29)}$ is significantly higher than that of $\operatorname{DPAB}\left(29^{\circ} \mathrm{C}\right)^{32)}$, which could be related to their respective abilities to increase fluidity.

To elucidate the effect of the alkyl-chain length of the dialkylammonium cationic surfactants, we performed DPH anisotropy experiments with dilauryl(DLAC), dimyristyl (DMAB), dipalmityl (DPAB), and distearyl ammonium (DSAC). As expected, the reduction of the anisotropy occurred in the following order: $\mathrm{DLAC}>\mathrm{DMAB}>\mathrm{DPAB}>\mathrm{DSAC}$ (Fig. 5). This result suggests that dialkylammonium surfactants with shorter alkyl chains (and thus a lower $\left.T_{\mathrm{m}}\right)^{32-37)}$ more efficiently increase the membrane fluidity of the liposome.

\subsection{Emission spectra of Laurdan in eggPC/eggSM/cho- lesterol liposome.}

We also evaluated the membrane fluidity using a microenvironment-sensitive probe, i.e., Laurdan. The fluorescence emission spectra of Laurdan are highly dependent upon the phase state of the lipid matrix. Laurdan emission spectra undergo bathochromic shifts as the lipid matrix converts from $\mathrm{L}_{\mathrm{o}}$ to $\mathrm{L}_{\mathrm{d}}{ }^{20-24)}$. Figure 6 shows the changes in the emission spectra of Laurdan in eggPC/eggSM/cholesterol liposome after the addition of DLAC or ethyl-DLPC.
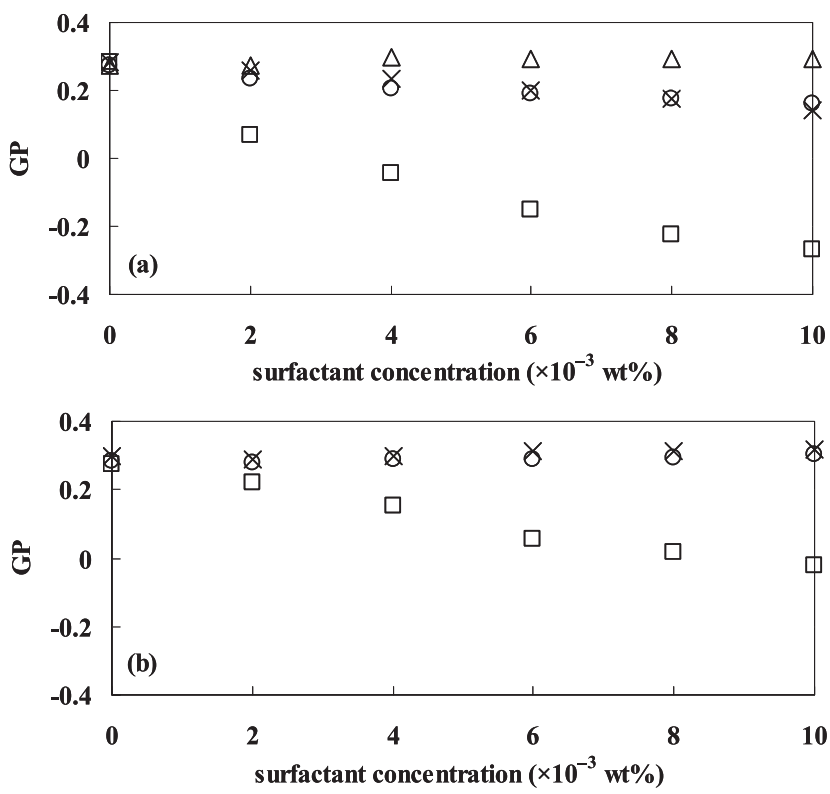

Fig. 7 Changes in GP values as a function of the wt $\%$ concentration of surfactants added. The surfactants in panel(a) are DLPA (triangles), ethylDLPC (circles), DLPC (crosses), and DLAC (squares), and the surfactants in panel (b) are DHP (crosses), DPPC (circles), and DPAB (squares). 
Upon the addition of DLAC, the emission maxima of the spectra red-shifted from 440 to $490 \mathrm{~nm}$, which suggests the phase transition from $\mathrm{L}_{\mathrm{o}}$ to $\mathrm{L}_{\mathrm{d}}$. In contrast, there was little change in the emission spectra after the addition of ethylDLPC, which suggests that the phase transition does not occur. The shift of the emission maximum was quantified by the GP value using Eq. (2). The results are shown in Figs. $7(\mathrm{a})$ and (b). Similarly to the results for DPH anisotropy, the GP values revealed that the membrane fluidity of liposome only increased significantly when dialkylammonium-type cationic surfactants were added. Since the minimum GP value in the presence of DLAC (approximate-

(A)
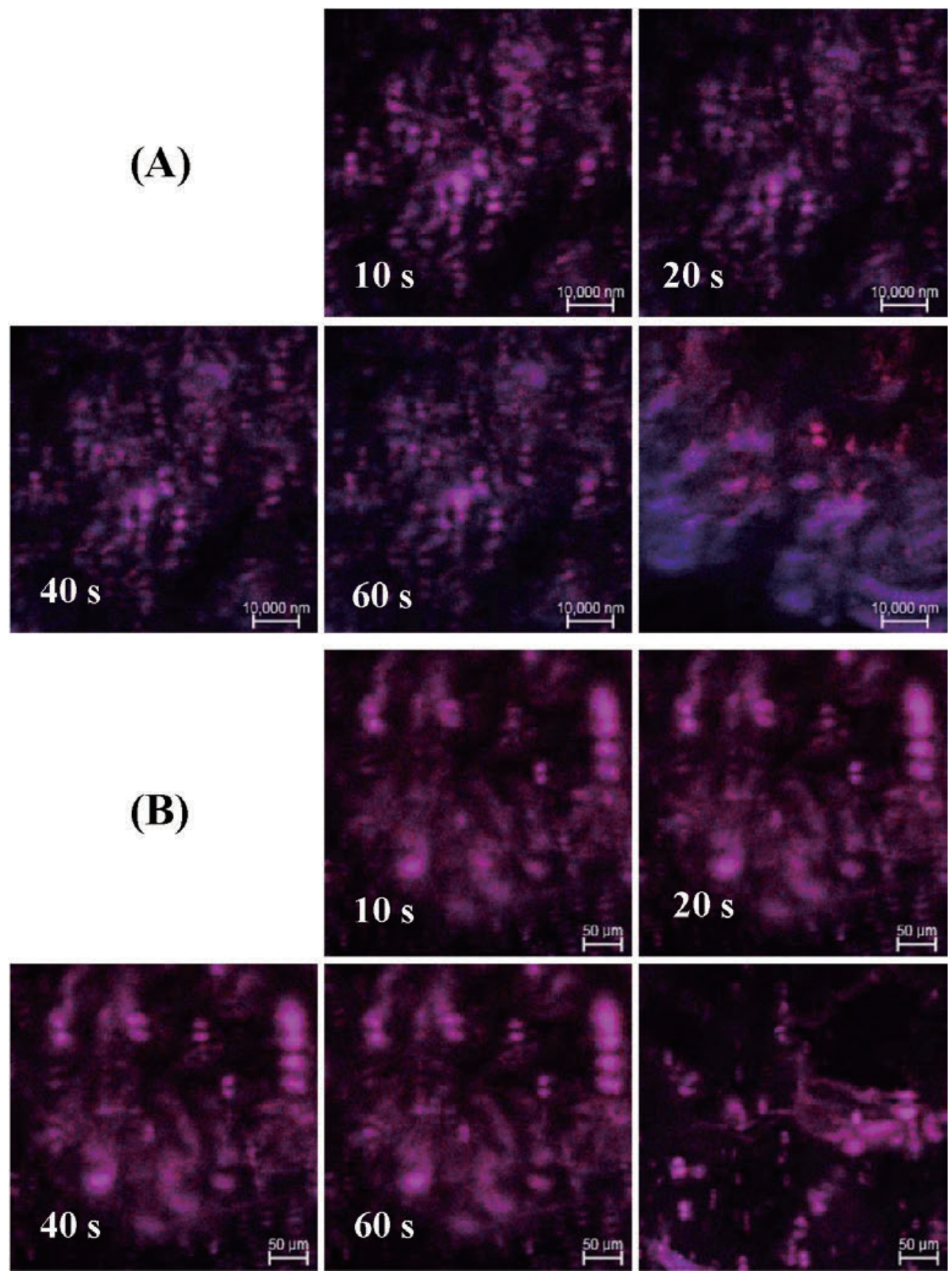

Fig. 8 Two-photon excitation microscopy merge images of the effects of DLAC (panel A) and DLPC (panel B) on Laurdan in the DPPC bilayer membrane at $30^{\circ} \mathrm{C}$. Red and blue represent the Laurdan fluorescence at 440 and $490 \mathrm{~nm}$, respectively. The times indicated in the figures denote the time elapsed(s)after the addition of DLAC or DLPC. The lower-right corner of the image in each panel shows the image with a different focal length and scope, $60 \mathrm{~s}$ after the addition of DLAC or DLPC. 


\section{Uyama, K. Inoue, K. Kinoshita et al.}

ly -0.3) is the value commonly observed for membranes in the liquid-crystalline phase ${ }^{21}$, the raft regions are considered to entirely convert to the $\mathrm{L}_{\mathrm{d}}$ phase.

\subsection{Two-photon excitation microscopy images}

Since a multi-lamellar membrane consisting of eggPC/ eggSM/cholesterol was difficult to obtain on a slide glass due to the deposition of cholesterol crystals, a DPPC membrane was used for two-photon microscopy. Since the $T_{\mathrm{m}}$ of DPPC is about $41^{\circ} \mathrm{C}^{38)}$, the DPPC membrane is considered to be in the gel state in this experiment $\left(30^{\circ} \mathrm{C}\right)$. Figure 8 shows two-photon excitation microscopy images of Laurdan in a DPPC multi-bilayer membrane several seconds after the addition of DLAC or DLPC. The red and

(A)
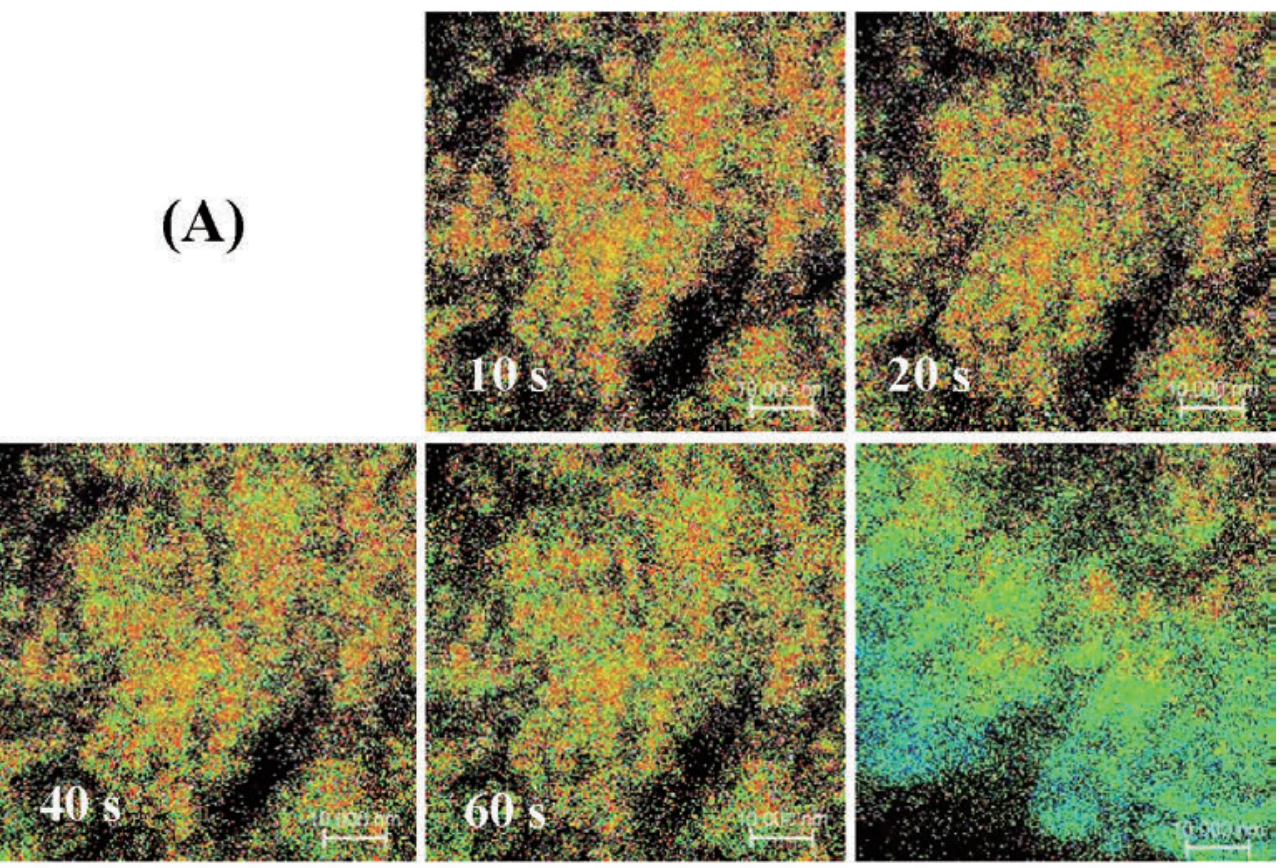

(B)
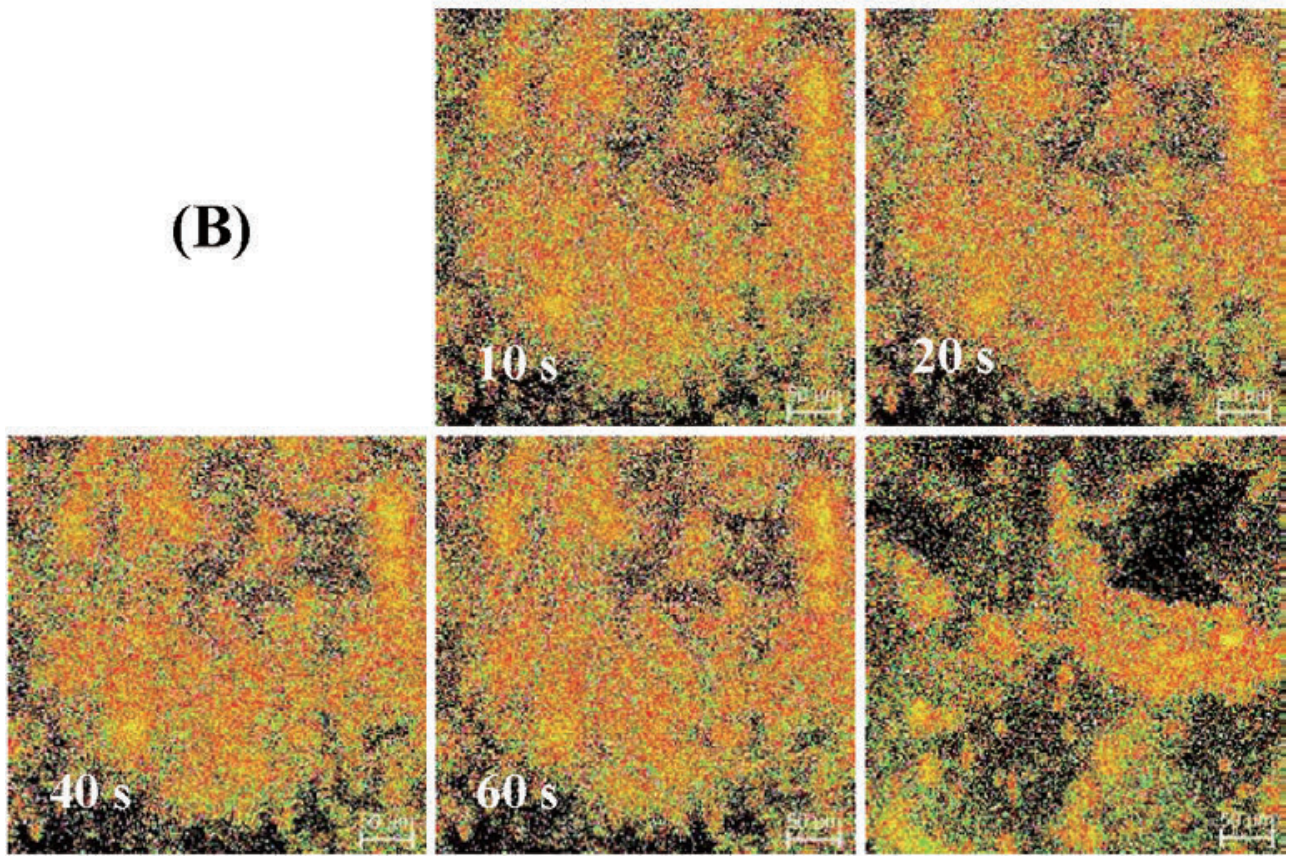

Fig. 9 Two-photon excitation microscopy GP images corresponding to the images in Fig. 8. The GP images were obtained by applying the GP function to the images from channel-1 and -2 on a per pixel basis. Blue and red represent the regions with the lowest and highest values of GP. The lower-right corner of the image in each panel shows the image at a different focal length and scope, $60 \mathrm{~s}$ after the addition of DLAC or DLPC. 
blue represent Laurdan fluorescence at 440 and $490 \mathrm{~nm}$, respectively. The Laurdan fluorescence at $490 \mathrm{~nm}$ increased in intensity upon the addition of DLAC, but was hardly affected by the addition of DLPC. Figure 9 shows the corresponding GP images that were calculated using Eq. (2); the regions with the lowest and highest GP values are colored blue and red, respectively. In Fig. $9(\mathbf{A})$, the red and blue regions gradually decreased and increased, respectively, after the addition of DLAC, which suggests that DLAC promptly inserts into the DPPC bilayers and increases the membrane fluidity. This result provides evidence of the effective insertion of DLAC into stiff bilayers in the gel phase and likely rafts as well.

\section{CONCLUSION}

The experimental results support several conclusions: (1) The electrostatic interaction between the cationic surfactant and eggPC/eggSM/cholesterol liposome could be important. Unlike anionic groups, the cationic head-group of the surfactants would tend to localize in a deeper region of the membrane in order to interact with the phosphate group of the phospholipids. Reorientation of the $\mathrm{P}^{-}-\mathrm{N}^{+}$ dipoles of the phosphocholine head-group by cationic surfactants could occur and affect the fluidity ${ }^{32,39)}$. (2) Surfactants with alkyl chains, such as DLAC, more effectively inserted into membranes than those with acyl chains, such as ethyl-DLPC. (3) Cationic surfactants with lower $T_{\mathrm{m}}$ values have a greater ability to increase the fluidity. Among the surfactants used, DLAC fulfills all of these conditions. In addition, the van der Waals interaction between hydrocarbon chains as well as the electrostatic interaction between polar head groups is also important. The cationic surfactants used in this study have saturated alkyl chains and therefore have high affinity with sphingomyelin and DPPC which have saturated acyl chains, resulting that these cationic surfactants interact with the raft membrane ( $\mathrm{L}_{0}$ phase). When DLAC, which has short alkyl chains, is incorporated into the raft domain, defects in lipid packing would arise. The membrane fluidity was increased due to the defects. On the other hand, Heerklotz reported that a small amount of a membrane-disordering additive(Triton) greatly enhanced domain formation by a rather unspecific mechanism ${ }^{40)}$. The role of hydrophilic head groups and that of hydrophobic groups must be very important for the raft formation but remain poorly understood ${ }^{41}$. The membrane curvature may affect the formation and stability of lipid rafts $^{42-45}$. Liposomes are used as a model membrane in this study, however, the size of liposomes is much smaller than that of living cells, therefore curvature and lipid composition may be greatly different from those of cell membranes. However, what is important is that we have found that among various surfactants a certain cationic surfactant increases the fluidity of the membrane by using liposomes. Since the evaluation of membrane fluidity with liposomes is simple, one can easily perform a screening test like in this study.

\section{References}

1) Morenilla-Palao, C.; Pertusa, M.; Meseguer, V.; Cabedo, H.; Viana, F. Lipid raft segration modulates TRPM8 channel Activity. J. Biol. Chem. 284, 9215-9224 (2009).

2) Baraldi, P.G.; Preti, D.; Materazzi, S.; Geppetti, P. Transient receptor potential ankyrin 1 (TRPA1) channel as emerging target for novel analgesics and anti-inflammatory agents. J. Med. Chem. 53, 5085-5107 (2010).

3) Bharate, S.S.; Bharate, S.B. Modulation of thermoreceptor TRPM8 by cooling compounds. ACS Chem. Neurosci. 3, 248-267(2012).

4) Bagal, S.K.; Brown, A.D.; Cox, P.J.; Omoto, K.; Owen, R.M.; Pryde, D.C.; Sidders, B.; Skerratt, S.E.; Stevens, E.B.; Storer, R.I.; Swain, N.A. Ion channels as therapeutic targets: a drug discovery perspective. J. Med. Chem. 56, 593-624(2013).

5) Ceacero-Vega, A.A.; Ballesteros, B.; Bejan, I.; Barnes, I.; Jimenez, E.; Albaladejo, J. Kinetics and mechanisms of the tropospheric reactions of menthol, borneol, fenchol, camphor, and fenchone with hydroxyl radicals (OH) and chlorine atoms (Cl). J. Phys. Chem. A 116, 4097-4107 (2012).

6) Szoke, E.; Börzsei, R.; Toth, D.M.; Lengl, O.; Helyes, Z.; Sandor, Z.; Szolcsanyi, J. Effect of lipid raft disruption on TRPVi receptor activation of trigeminal sensory neurons and transfected cell line. Eur. J. Pharmacol. 628, 67-74 (2010).

7) Janes, P.W.; Ley, S.C.; Magee, A.I.; Kabouridis, P.S. The role of lipid rafts in $\mathrm{T}$ cell antigen receptor(TCR) signaling. Semin. Immunol. 12, 23-34(2000).

8) Calzolari, A.; Raggi, C.; Deaglio, S.; Sposi, N.M.; Stafsnes, M.; Fecchi, K.; Parolini, I.; Malavasi, F.; Peschle, C.; Sargiacomo, M.; Testa, U. TfR2 localizes in lipid raft domains and is released in exosomes to activate signal transduction along the MAPK pathway. $J$. Cell Sci. 119, 4486-4498(2006).

9) Quinn, P.J.; Wolf, C. Egg-sphingomyelin and cholesterol form a stoichiometric molecular complex in bilayers of egg-phosphatidylcholine. J. Phys. Chem. B 114, 15536-15545 (2010).

10) De Almeida, R.F.M.; Fedorov, A.; Prieto, M. Sphingomyelin/phosphatidylcholine/cholesterol phase diagram: boundaries and composition of lipid rafts. Biophys. J. 85, 2406-2416(2003).

11) Fukuda, M.; Nakano, M.; Sriwongsitanont, S.; Ueno, M.; Kuroda, Y.; Handa, T. Spontaneous reconstitution of 


\section{Uyama, K. Inoue, K. Kinoshita et al.}

discoidal HDL from sphingomyelin-containing model membranes by apolipoprotein A-I. J. Lipid Res. 48, 882-889 (2007).

12) $\mathrm{Xu}, \mathrm{X}$; London, E. The effect of sterol structure on membrane lipid domains reveals how cholesterol can induce lipid domain formation. Biochemistry 39, 843849 (2000).

13) Saito, H.; Nishiwaki, K.; Handa, T.; Ito, S.; Miyajima, K. Comparative study of fluorescence anisotropy in surface monolayers of emulsions and bilayers of vesicles. Langmuir 11, 3742-3747 (1995).

14) Kaiser, R.D.; London, E. Location of diphenylhexatriene (DPH) and its derivatives within membranes: comparison of different fluorescence quenching analysis of membrane depth. Biochemistry 37, 8180-8190 (1998).

15) Raghuraman, H.; Pradhan, S.K.; Chattopadhyay, A. Effect of urea on the organization and dynamics of Triton X-100 micelles: a fluorescence approach. J. Phys. Chem. B 108, 2489-2496 (2004).

16) Zana, R.; In, M.; Levy, H. Alkanediyl- $\alpha, \omega$-bis (dimethylalkylammonium bromide). 7. fluorescence probing studies of micelle micropolarity and microviscosity. Langmuir 13, 5552-5557 (1997).

17) Venegas, B.; Sugar, I.P.; Chong, P.L. Critical factors for detection of biphasic changes in membrane properties at specific sterol mole fractions for maximal superlattice formation. J. Phys. Chem. B 111, 5180-5192 (2007).

18) Lönnfors, M.; Engberg, O.; Peterson, B.R.; Slotte, J.P. Interaction of $3 \beta$-amino- 5 -cholestene with phospholipids in binary and ternary bilayer membranes. Langmuir 28, 648-655 (2012).

19) Esquembre, R.; Ferrer, M.L.; Gutierrez, M.C.; Mallavia, R.; Mateo, C.R. Fluorescence study of the fluidity and cooperativity of the phase transitions of zwitterionic and anionic liposomes confined in sol-gel glasses. $J$. Phys. Chem. B 111, 3665-3673(2007).

20) Massey, J.B.; Pownall, H.J. The polar nature of 7-ketocholesterol determines its location within membrane domains and the kinetics of membrane microsolubilization by apolipoprotein A-I. Biochemistry 44, 1042310433 (2005).

21) Sanchez, S.A.; Tricerri, M.A.; Gunther, G.; Gratton, E. Laurdan generalized polarization: from cuvette to microscope. Modern Research and Educational Topics in Microscopy. Applications in Physical/Chemical Sciences 1007-1014(2007).

22) Best, K.B.; Ohran, A.J.; Hawes, A.C.; Hazlett, T.L.; Gratton, E.; Judd, A.M.; Bell, J.D. Relationship between erythrocyte membrane phase properties and susceptibility to secretory Phospholipase $\mathrm{A}_{2}$. Biochemistry 41, 13982-13988(2002).

23) Esquembre, R.; Poveda, J.A.; Mateo, C.R. Biophysical and functional characterization of an ion channel peptide confined in a sol-gel matrix. J. Phys. Chem. B 113, 7534-7540 (2009).

24) Rowe, B.A.; Neal, S.L. Photokinetic analysis of PRODAN and LAURDAN in large unilamellar vesicles from multivariate frequency-domain fluorescence. J. Phys. Chem. B 110, 15021-15028(2006).

25) Hughes, D.J.; Cui, Z.; Field, R.W.; Tirlapur, U.K. In situ three-dimensional characterization of membrane fouling by protein suspensions using multiphoton microscopy. Langmuir 22, 6266-6272(2006).

26) Tsogas, I.; Tsiourvas, D.; Nounesis, G.; Paleos, C.M. Interaction of poly-L-argininge with dihexadecyl phosphate/phosphatidylcholine liposomes. Langmuir 21, 5997-6001 (2005).

27) Kurshev, V.; Kevan, L. Comparison photoelectron transfer between $\mathrm{Ru}(\text { bpy })_{3}{ }^{2+}$ and $\mathrm{MV}^{2+}$ in $\mathrm{TiO}_{2}$ and $\mathrm{SnO}_{2}$ colloids and dihexadecyl phosphate vesicles. Langmuir 13, 225-228(1997).

28) Khairutdinov, R.F.; Hurst, J.K. Photophysical deactivation dynamics of excited porphyrin molecules adsorbed onto dihexadecyl phosphate vesicles. J. Phys. Chem. B 103, 3682-3686(1999).

29) Thünemann, A.F.; Kurth, D.G.; Beinhoff, M.; Bienert, R.; Schulz, B. V-shapped crystalline structures of di- $n$ alkyl esters of phosphoric acid. Langmuir 22, 58565861 (2006).

30) Rupert, L.A.M.; Engberts, J.B.F.N.; Hoekstra, D. Effect of poly (ethylene glycol) on the $\mathrm{Ca}^{2+}$-induced fusion of didodecyl phosphate vesicles. Biochemistry 27, 82328239 (1988).

31) Rupert, L.A.M.; van Breemen, J.F.L.; Hoekstra, D.; Engberts, J.B.F.N. pH-Dependent fusion of didodecyl phosphate vesicles. Role of hydrogen-bond formation and membrane fluidity. J. Phys. Chem. 92, 4416-4420 (1988).

32) Ryhänen, S.J.; Alakoskela, J-M.I. Kinnunen, P.K. Increasing surface charge density induces interdigitation in vesicles of cationic amphiphile and phosphatidylcholine. Langmuir 21, 5707-5715(2005).

33) Kunieda H.; Shinoda, K. Solution behavior of dialkyldimethylammonium chloride in water. Basic properties of antistatic fabric softeners. J. Phys. Chem. 82, 17101714 (1978).

34) Marques, E.F.; Regev, O.; Khan, A.; Lindman, B. Selforganization of double-chained and pseudodoublechained surfactants: counterion and geometry effects. Adv. Collid Interface Sci. 100-102, 83-104(2003).

35) Laughlin, R.G.; Munyon, R.L.; Fu, Y.C.; Emge, T.J. Physical science of the dioctadecyldimethylammonium chloride-water system. 2. Kinetic and mechanistic aspects. J. Phys. Chem. 95, 3852-3856(1991).

36) Caboi, F.; Monduzzi, M. Didodecyldimethylammonium bromide vesicles and lamellar liquid crystals. A multi- 
nuclear NMR and optical microscopy study. Langmuir 12, 3548-3556 (1996).

37) Kano, K.; Romero, A. Djermouni, B.; Ache, H.J.; Fendler, J.H. Characterization of surfactant vesicles as membrane mimetic agents. 2. Temperature-dependent changes of the turbidity, viscosity, fluorescence polarization of 2-methylanthracene, and positron annihilation in sonicated dioctadecyldimethylammonium chloride. J. Am. Chem. Soc. 101, 4030-4037(1979).

38) Cherney, D.P.; Conboy, J.C.; Harris, J.M. Optical-trapping Raman microscopy detection of single unilamellar lipid vesicles. Anal. Chem. 75, 6621-6628(2003).

39) Oliveira, T.R.; Duarte, E.L.; Lamy, M.T.; Vandenbranden, M.; Ruysschaert, J-M.; Lonez, C. Temperature-dependence of cationic lipid bilayer intermixing: possible role of interdigitation. Langmuir 28, 4640-4647 (2012).
40) Heerklotz, H. Triton promotes domain formation in lipid raft mixtures. Biophys. J. 83, 2693-2701 (2002).

41) London, E. Insights into lipid raft structure and formation from experiments in model membranes. Curr. Opin. Struct. Biol. 12, 480-486(2002).

42) van Meer, G.; Vaz, W.L.C. Membrane curvature sorts lipids. EMBO Rep. 6, 418-419(2005).

43) Feigenson, G.W. Phase behavior of lipid mixtures. Nat. Chem. Biol. 2, 560-563(2006).

44) Goni, F.M.; Alonso, A.; Bagatolli, L.A.; Brown, R.E.; Marsh, D.; Prieto, M. Thewalt, J.L. Phase diagrams of lipid mixtures relevant to the study of membrane rafts. Biochim. Biophys. Acta 1781, 665-684(2008).

45) Sadeghi, S.; Müller, M.; Vink, R.L.C. Raft formation in lipid bilayers coupled to curvature. Biophys. J. 107, 1591-1600(2014). 